\title{
Overlapping Grid Technique for Numerical Simulation of a Fast-Cruising Catamaran Fitted with Active T-Foils
}

\author{
Dongmei Yang ${ }^{1} \cdot$ Fei Shao ${ }^{2} \cdot$ Chuanglan $\mathrm{Li}^{1} \cdot$ Hongqing Chen ${ }^{1}$
}

Received: 27 March 2017 / Accepted: 18 November 2018/Published online: 4 March 2019

(C) The Author(s) 2019

\begin{abstract}
In marine engineering, appendages such as fin stabilizers and/or T-foils are made to rotate and to reduce the motion of ships. Research on the hydrodynamics of ships fitted with active appendages has significantly improved the design and control of such ships. However, most studies focus on fixed rather than rotating appendages, thereby ignoring the hydrodynamic unsteadiness of active appendages. To enhance the reliability and precision of the numerical simulations, we propose the use of overlapping grids for simulating advanced catamarans fitted with a pair of rotating T-foils under each bow. The fundamental purpose of the overlapping grid technique is to realize information exchange via regional overlap sharing in each subdomain of the computing domain, instead of using the method of boundary sharing, thus greatly alleviating the difficulty of generating the subdomain grid; moreover, the technique guarantees the quality of the subdomain grid. Within the main computational domain, a subdomain was allocated to accommodate the T-foil. Overlapping meshes near the interface between the two domains enable information flow during the simulation; the overlapping grids are updated at every iteration step because the subdomain rotates. The instantaneous trim and sinkage responses of the catamaran to the T-foil rotation were reproduced. From the moment the active T-foil stopped moving, there was no change in the ship's sailing attitude, indicating that the response was in real time. By comparing with EFD data, the numerical results showed reasonable agreement, indicating the feasibility and effectiveness of the technique in simulating the hydrodynamics of ships fitted with active appendages.
\end{abstract}

Keywords Overlapping grids $\cdot$ Numerical simulation · Active appendages $\cdot$ T-foil $\cdot$ Catamaran

\section{Introduction}

With the flourish of high-speed ships in recent years, maintaining effective seakeeping under high-cruise velocity and rough conditions has become a critical issue. Matching fin stabilizers and T-foils to hulls has been widely proposed to realize this. Some appendages actively work in the water to

\section{Article Highlights}

- The overlapping grid technique is used to simulate the hydrodynamic characteristics of the T-foil.

- The numerical results show reasonable agreement with experimental data.

Dongmei Yang

yangdm411@126.com

1 College of Shipbuilding Engineering, Harbin Engineering University, Harbin 150001, China

2 China Ship Development and Design Center, Wuhan 430064, China provide a stronger ability to improve the seakeeping of highcruising ships. In this work, we focus on a high-speed catamaran equipped with a pair of T-foils under the bow of each semi-hull. Ji et al. (2006) used T-foils to reduce the longitudinal motion of the hull. The function of foils is to increase the added mass and change hydrodynamic lift damping (Yang et al. 2017; Jiang et al. 2016; Liu et al. 2016; Liu 2016; Jin et al. 2015; Si et al. 2015; Liu 2014; Ma 2012; Shao and Wang 1981). Assisted by a control system, active T-foils can be suitably rotated in real time to counteract excessive trim or sinkage much better than fixed foils.

The hydrodynamic characteristics of T-foils have been investigated in many studies. Esteban et al. (2005) and Liang et al. (2013) evaluated the lift coefficient by deducing an empirical expression obtained using linear regression from experimental results. The results might have limitations if the influence of the free surfaces is not considered, as such influence has been confirmed to be significant in experiments when the T-foil is close to a free surface (Ning 2017; Wang 2017; Liang 2015; Yang 2012; Chen et al. 2010). In fact, the 
closer the T-foil is to the free surface, the smaller the lift is. Yang et al. (2013) used the panel method to calculate the hydrodynamics of a T-foil under the bow of a trimaran, where the effects of both free surfaces and hulls were considered. The investigation results showed that the lift increased as the T-foil approached the hull surface, in contrast to a free surface. However, in Yang's work, the running attitude of the trimaran was presumed, and viscous effects of the T-foil and hulls were still not considered. Deng et al. (2014) used the approach of Reynold-averaged Navier-Stokes equations to perform numerical simulations of a trimaran matched with a fixed T-foil. They concluded that the foil can improve seakeeping ability but slightly increases navigation drag. However, under certain conditions, the unsteady hydrodynamics of active T-foils might be significant, leading to a difference from those of fixed T-foils. Hao et al. showed that if the Strouhal number of the foil is beyond a certain threshold, the rotational foil can produce averaged positive thrust in flows (Hao et al. 2015). One can imagine that if steady methods are used to analyze the hydrodynamics of foils, the thrust will always be negative in the presence of drag. Unfortunately, Deng's meshing method cannot be employed for simulating active T-foils, because meshes between the hull and T-foil are not independent. Once the T-foil boundary moves, an operation error will occur in the iterative computation process.

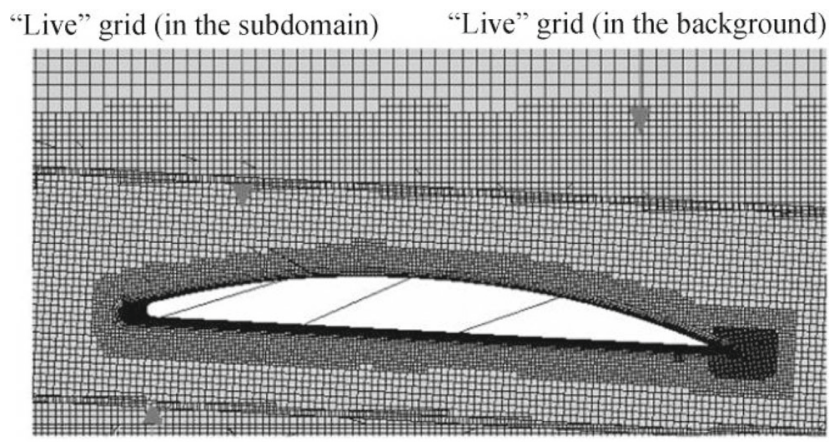

Overlapping band

(a) Initial mesh

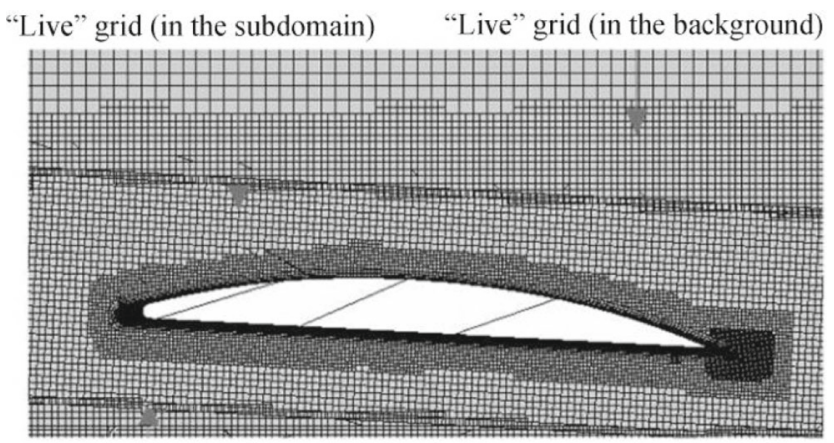

Overlapping band

(b) Mesh after digging a hole

Fig. 1 Digging process. a Initial mesh. b Mesh after digging a hole
Based on the viscous flow theory, an overlapping grid technique is proposed in this study to numerically simulate the motion of a catamaran fitted with a pair of T-foils. Because the model is symmetrical, only one semi-hull and T-foil are generated and simulated. The key point is to set up two domains in the flow field that establish a background comprising the main body and a subdomain containing the T-foil. In each domain, a meshing is generated, and the two meshes overlap across the interface between the subdomain and the background field. The overlapping grids typically consist of three or four layers. The flow information between the subdomain and the background field is exchanged through receiving and transmitting grids in the overlapping layers. With the rotation of the subdomain, overlapping grids are updated at each iteration step. As a result, one can obtain the sinkage and trim response, as well as the variation in the navigation resistance to the flapping T-foil.

\section{Theory of Numerical Simulation}

The finite volume-based STAR-CCM+ software is used to solve the fluid field around the after body. The governing equations of the incompressible viscous fluid are described by the Reynold-averaged Navier-Stokes and continuity equations. To close this set of equations, the shear stress transport (SST) $k$ - $\omega$ turbulence model is used since it accounts for the transport of the turbulent shear stress and gives highly accurate predictions of the onset and the amount of flow separation under adverse pressure gradients. The governing equation of the flow field and mathematical expression of SST $k-\omega$ turbulence mode are described below:

$\frac{\partial p}{\partial t}+\frac{\partial\left(\rho u_{i}\right)}{x_{i}}=0$

$\frac{\partial\left(\rho u_{i}\right)}{\partial t}+\frac{\partial\left(\rho u_{i} u_{j}\right)}{x_{i}}=-\frac{\partial p}{\partial x_{i}}+\frac{\partial}{x_{j}}\left(\mu \frac{\partial u_{i}}{\partial x_{j}}-\rho \overline{u_{i}^{\prime} u_{j}^{\prime}}\right)+S_{i}$

where $u_{i}$ and $u_{j}$ are the time average of velocity component ( $i$, $j=1,2,3) ; p$ is the time average of pressure; $\rho$ is the fluid density of water at $20^{\circ}\left(998.16 \mathrm{~kg} / \mathrm{m}^{3}\right) ; \mu$ represents the dynamic viscosity coefficient of water $(0.001003 \mathrm{~kg} / \mathrm{ms})$; $\rho u_{i}^{\prime} u_{j}^{\prime}$ and $S_{i}$ are the Reynolds stress term and volumetric force, respectively.

The transport equation of $k$ is as follows:

$$
\frac{\partial}{\partial t}(\rho k)+\frac{\partial}{\partial x_{i}}\left(\rho k u_{i}\right)=\frac{\partial}{\partial x_{i}}\left(\Gamma_{k} \frac{\partial k}{\partial x_{i}}\right)+G_{k}-Y_{k}
$$

The transport equation of $\omega$ is as follows:

$$
\frac{\partial}{\partial t}(\rho \omega)+\frac{\partial}{\partial x_{i}}\left(\rho \omega u_{i}\right)=\frac{\partial}{\partial x_{i}}\left(\Gamma_{\omega} \frac{\partial \omega}{\partial x_{i}}\right)+G_{\omega}-Y_{\omega}+D \omega
$$




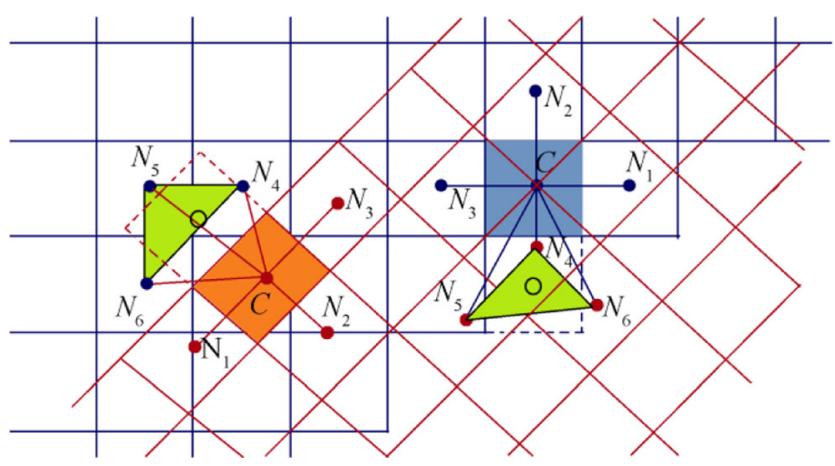

Fig. 2 Transmission of variables

where $G_{k}$ and $G_{\omega}$ are turbulent kinetic energies due to the average velocity gradient; $Y_{k}$ and $Y_{\omega}$ are turbulent dissipation terms.

\section{Overlapping Grid Technique}

The basic purpose of the overlapping grid technique is to realize information exchange using the method of regional overlap sharing in each subdomain of the computing domain, instead of using the method of boundary sharing, thus greatly alleviating the difficulty of generating the subdomain grid; moreover, the technique guarantees the quality of the subdomain grid. Therefore, this technique can be applied for simulating a numerical model with complex shapes. It has many advantages, such as high precision of flow field calculation, high efficiency, and strong wall stickiness simulation ability, which make up for the disadvantage of poor shape adaptability of grid structure.

The overlapping grid technique requires dividing the computing domain into two or several subdomains in the calculated fluid field, and then the moving boundary can be simulated using the motion of the subdomains (Kehoe et al. 1983). These subdomains and background domain can be overlapped by any means, and these domains can exchange information through the overlapping grids.

The subdomain contains the entire moving body. Normally, the number of independent relative motions of the moving body dictates the number of subdomains. The boundary shape is determined by the specific subdomain boundary shape and motion of the moving body. In this case, the subdomains and the background field are independent of each other.

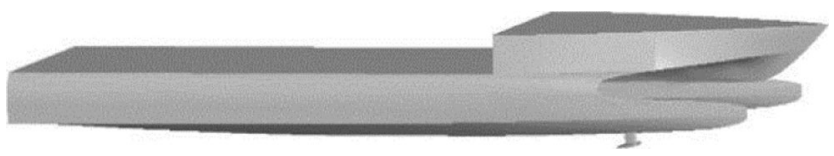

Fig. 3 3D model of ship and T-foil
The subdomain and background domain are coupled via the outer boundary, i.e., the overlapping part of subdomain and background domain, and thus, the coupled areas become a virtual boundary that acts as an interface. In each subdomain and background domain, the grid is independently generated. After the coupling, a portion of the background grid is "dug." As shown in Fig. 1. In this process, the grid status is determined. The grid is usually divided into an "active" grid, "inactive" grid, "interpolated" grid, and "transfer" grid. In the "active" grid, we need to solve the equations of the grid mesh on the grid nodes. In the "inactive" grid, there are no equations to be solved, and in the "digging" process, the "inactive" grid will be dug. Usually the "dead" grid is the part inside the subdomain boundary in the background domain. There is a strip through the overlapping area on the interface boundary. This area consists of the three- or four-level grid, only where the grid is overlapping. The "interpolated" and "transfer" grids are inside the overlapping band. The "interpolated" grid in the background domain separates the "active" grid from the "inactive" grid. In the subdomain, the "interpolated" grid is associated with the boundary grid. Variables are transmitted from the "transfer" grid to the "interpolated" grid via interpolation (Zhang et al. 2007).

There are several methods for realizing the interpolation, such as distance weighted interpolation, linear interpolation, and least squares interpolation. Figure 2 illustrates the way variable values are transmitted (Feng et al. 2008). An interpolation function is established in the coefficient matrix of the algebraic equations to ensure that the overlapping grid is implicitly coupled. Each "interpolated" grid, the center of which is marked with a small hollow circle, is picked out successively from the background domain and subdomain. The flux between the "active" grid and the nearest "interpolated" grid is almost the same as between the two adjacent "active" grids. However, as long as the "interpolated" variable value at the center of the grid is used, the weighted variable value is replaced by

$\phi$ "received"grid $=\sum \partial_{i} \phi_{i}$

where $\partial_{i}$ is the weighting factor for the interpolation, and $\phi$ is an independent variable value at the "transfer" grid $N_{i}$; subscript $i$ contains the entire "transfer" grid center in the interpolation element (shown as a triangular link). In this case, the algebraic equations involve the three adjacent grids $N_{1}, N_{2}$, and $N_{3}$ in the same region and the nearest grids $N_{4}, N_{2}$, and $N_{6}$ in the overlapping band from the other region.

Moreover, the segmented grid in this paper has adaptability characteristics. The adaptive grid is expected to be dense in the region with large physical solution, while the latent region of the material is relatively sparse, so that a high efficiency is obtained while maintaining high accuracy. Adaptive grid 
Fig. 4 Mesh generation

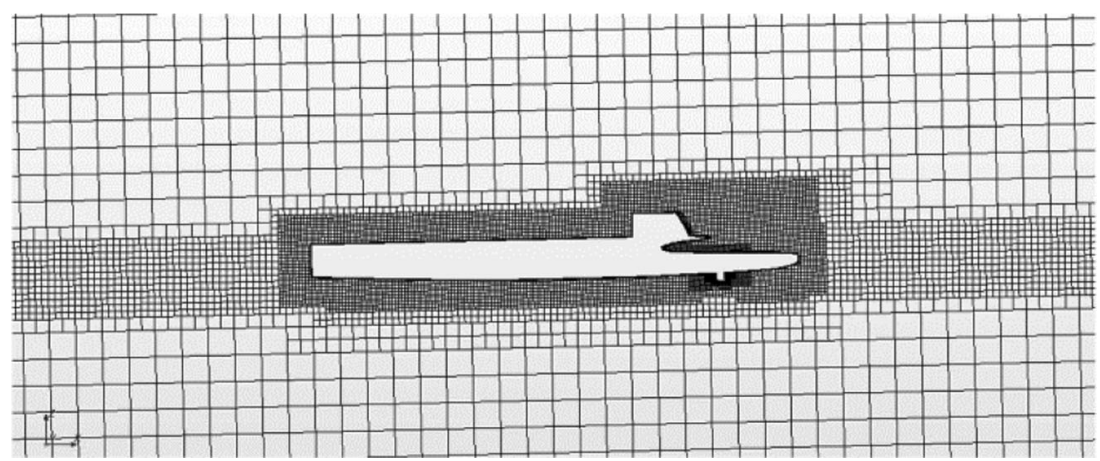

technology mainly includes a moving grid method and local refinement or coarsening of the grid method.

\section{Numerical Simulation of the T-Foil Hydrodynamics}

\subsection{Main Hull and T-Foil Models and Calculation Program}

In this study, a wave-piercing catamaran was modeled, with an active T-foil configured on the semi-hull. The distance between the centerline of the T-foil and the stern seal plate was $2.486 \mathrm{~m}$, and the dip deep of the T-foil was $60 \mathrm{~mm}$. After the meshing was completed, a multiphase flow model was built, using the volume-of-fluid model to capture the free surface. In fact, there was a long distance between the free surface and the T-foil, and the wave-making effect had no influence on the T-foil. The SST $k-\omega$ turbulence model and the first-order upwind scheme which are illustrated in the literature (Piperno 1997) were adopted for modeling. An implicit unsteady approach was used with a firstorder temporal discretization with a time step of $0.0025 \mathrm{~s}$. In the implicit unsteady approach, each physical time step involved 20 inner iterations to converge the solution for the given time step. The time step was limited based on the CourantFriedrichs-Lewy (CFL) number as given by Eq. (6).

$\mathrm{CFL}=\frac{U \Delta t}{\Delta x}$

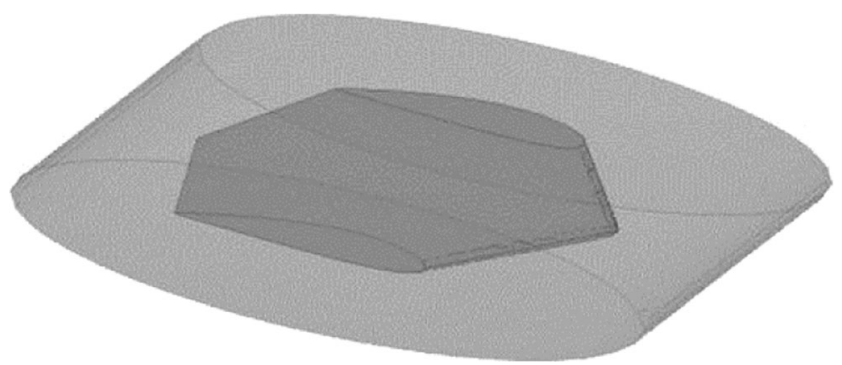

Fig. 5 Illustration of sub-region
The CFL defines a dimensionless relationship between the flow velocity $U$, local grid size, and the time step. The CFL number was maintained at less than 1 ; therefore, no more fluid could enter a given cell than was available in the upwind cell during each time step. It is possible for simulations with a larger CFL to produce solutions, but they will not be timeaccurate.

In the present work, the boundary conditions are specified as follows. At the inlet, the velocity (both for water and air) is

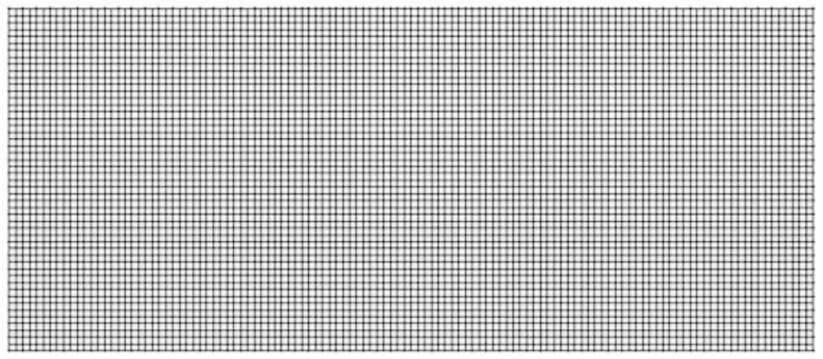

(a) Mesh for the main fluid region around T-foil

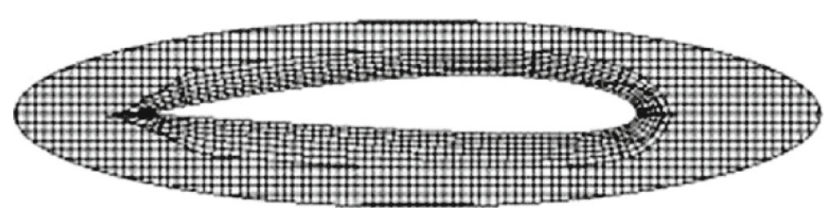

(b) Mesh for subdomain around the T-foil

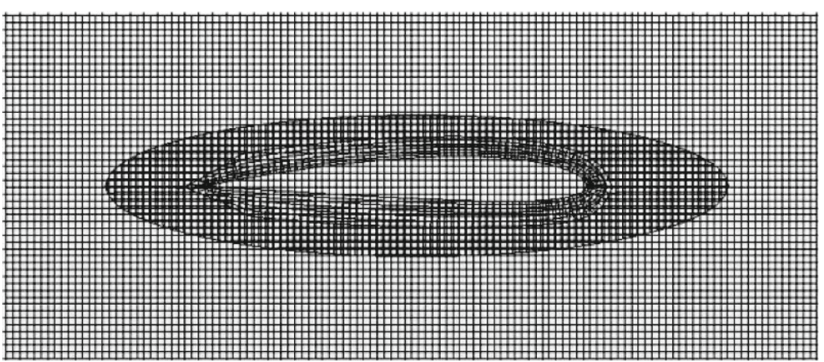

(c) Mesh after superoposition

Fig. 6 Mesh before and after superposition. a Mesh for the main fluid region around the T-foil. $\mathbf{b}$ Mesh for subdomain around the T-foil. $\mathbf{c}$ Mesh after superposition 


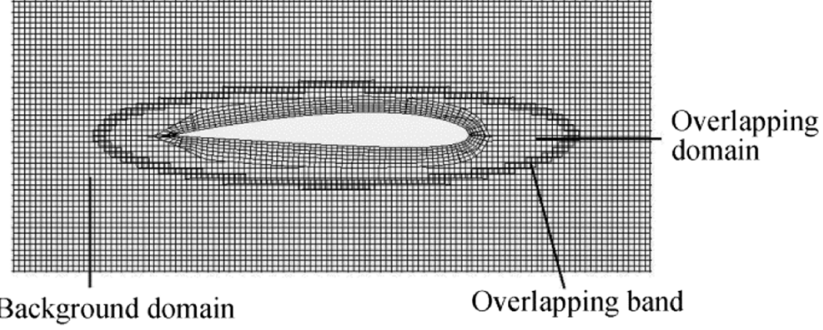

Fig. 7 Illustration of mesh after hole has been dug

specified by the moving forward speed of main hull and T-foil. At the outlet, hydrostatic pressure is applied. A symmetry condition is used at the longitudinal section in center plane for axial symmetry model. The model is considered a rigid body, and no-slip condition is imposed on main hull and Tfoil's surface. The 3D model of ship and T-foil are given in Fig 3 . And the mesh generation of the wave-piercing catamaran with T-foil is shown in Fig. 4.

The resolution of the mesh near the wall has significant effect on computational accuracy. In this study, 13 boundary layers were set to capture the flow near the wall, and the boundary layer mesh was refined with prism elements. Surface mesh size and non-dimensional wall distance of the first cell center $Y+$ were set to $0.006 L$ and 60 , respectively.

\subsection{Pretreatment of Grid and Domains in the Overlapping Grid}

The central idea of the overlapping grid method is to set up the two domains in the flow field, a background domain (main fluid domain) and a subdomain including the T-foil (Nirschl 2005). The subdomains are elliptical cylinders, and their cross section is shaped like a wing (Fig. 5).

Grids are generated independently for the two domains. Figure 6a shows the background grid for the background domain, near the T-foil but not containing it. The subdomain

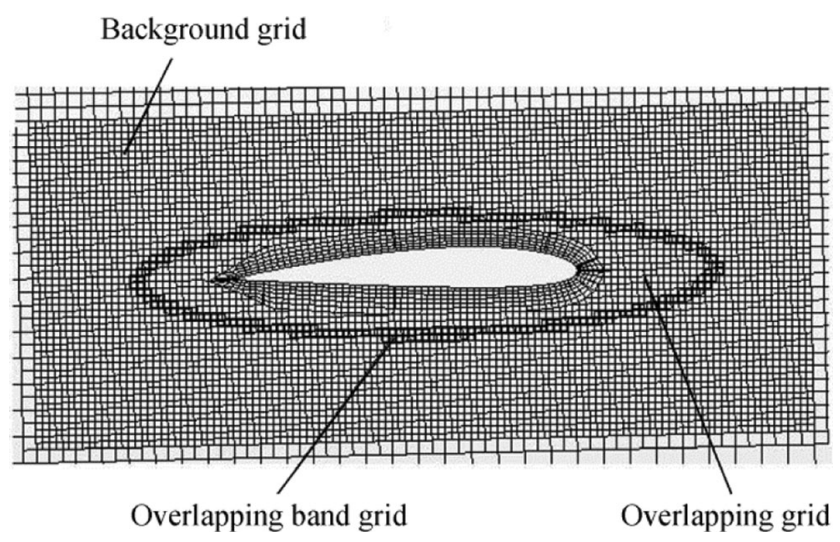

Fig. 8 Stage of sailing at $0^{\circ}$ angle of attack

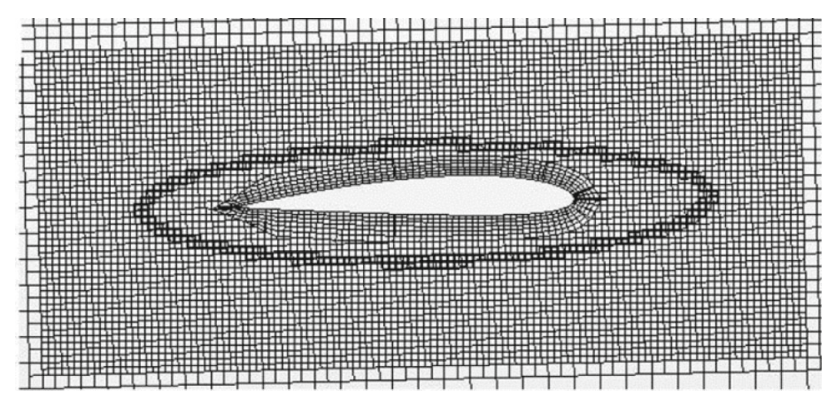

(a) At time $t_{0}$

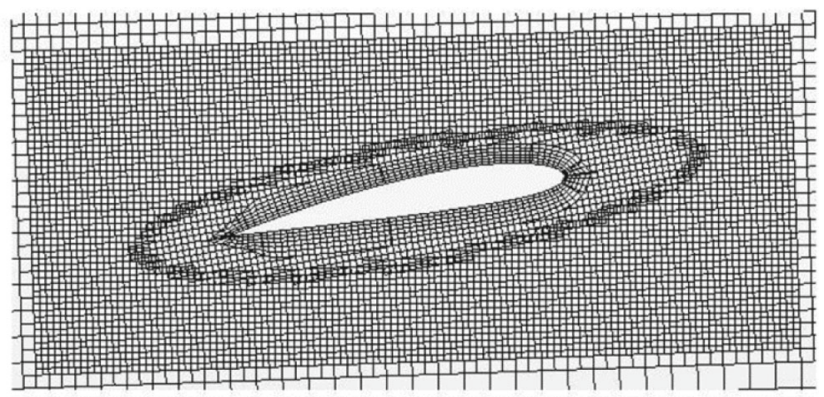

(b) At time $t_{1}$

Fig. 9 Stage of control (upswing): $0^{\circ}-8^{\circ}$. a At time $t_{0}$. b At time $t_{1}$

contains the T-foil; its meshing is shown in Fig. 6b. With the grids generated, the grid distribution of the flow field is finally obtained by superimposing the two grids (Fig. 6c).

The background grid is then "dug." All grids inside the oval-shaped domain of the background domain are

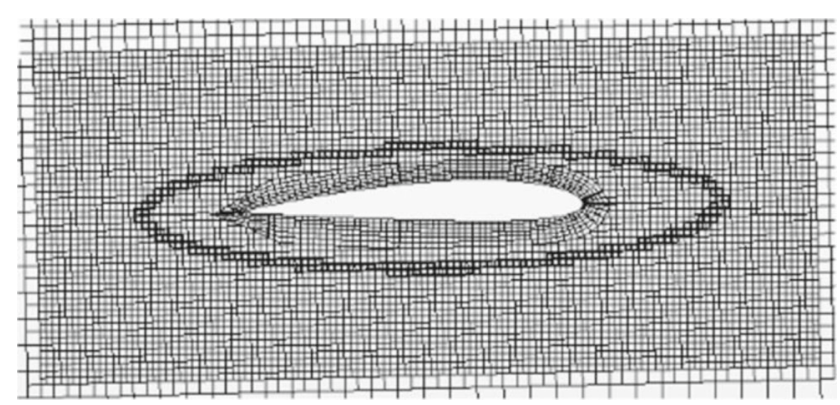

(a) At time $t_{0}$

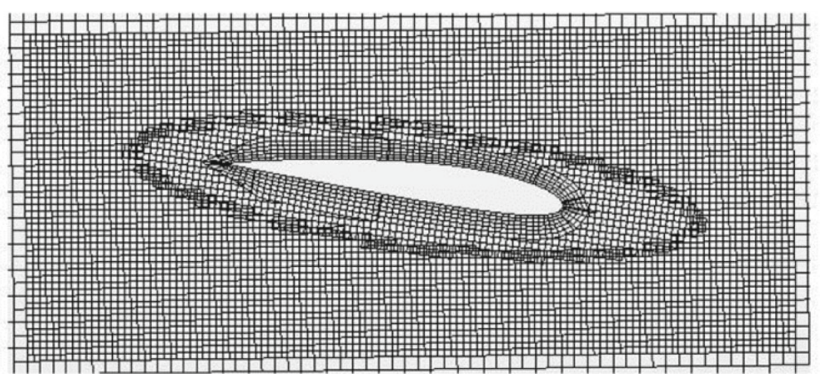

(b) At time $t_{1}$

Fig. 10 Stage of control (downswing): $0^{\circ}-8^{\circ}$. a At time $t_{0}$. b At time $t_{1}$ 


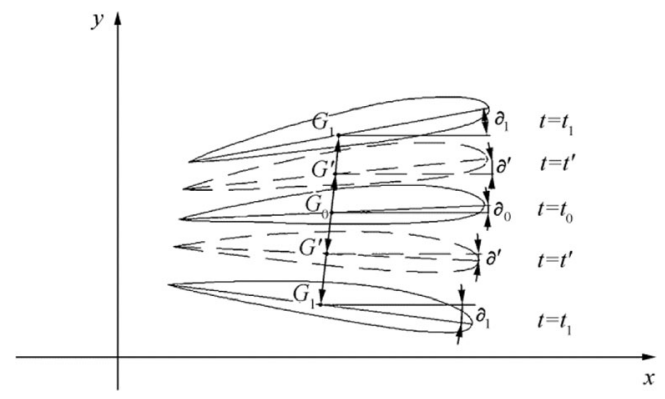

Fig. 11 Illustration of a swinging T-foil

overlapped with the subdomain grid. Using the "principle of minimum distance from the wall surface," the overlapping grid in the background grid is divided into a "live" grid and "dead" grid. The "dead" grid is then "dug." There will be a strip-shaped overlapping area on the interface boundary. This area consists of a three- or four-level grid, only where the grid is overlapping (Fig. 7). Information is exchanged between subdomain and background domain through the "receive" and "transfer" grids in the overlapping band. Note that when the subdomain moves, each iteration step will generate a new overlapping band.

\subsection{Active T-Foil Hydrodynamics Simulation Based on the Overlapping Grid}

The hydrodynamics simulation of the active T-foil can be divided into three stages:

\section{Sailing stage at the initial angle of attack}

In this stage, the T-foil-fitted catamaran sails at $4.115 \mathrm{~m} / \mathrm{s}$ with an initial angle of attack of $0^{\circ}$. Figure 8 illustrates the grid distribution around the T-foil after a period of time sailing at an initial angle of attack, which is set at $t_{0}$. At this time, the control system begins to control the T-foil actively.

\section{Active control stage (assembly motion stage):}

From the beginning of sailing, i.e., at time $t_{0}$, the active T-foil begins swinging, regulated by the control system, and stops swinging at time $t_{1}$ when the angle of attack is $8^{\circ}\left(\right.$ or $-8^{\circ}$ ). Figures 9 and 10 show changes in the grid when the angle of attack actively changes. In the overlapping grid zone, we can see a relative motion in the background grid, specifically, an active rotation of the T-foil. A closer inspection of the background grid shows that the background grid has some ups and downs and corners; this is because the moving computational domain method was chosen to model the hull.

This stage is the main stage in the simulation. To show the movement of the $\mathrm{T}$-foil more intuitively, in the stage from $t_{0}$ to $t_{1}$, the T-foil contour indicates changes in the plot of the geodetic coordinates (Fig. 11). Here $t^{\prime}$ denotes a moment from $t_{0}$ to $t_{1} ; G$ is the center of rotation of the T-foil and moves with the motion of the hull; $\partial$ is the angle between the direction of the T-foil chord and the $x$ direction. The time variation of $\partial$ is

$\partial(t)=\partial_{0} \pm \omega\left(t-t_{0}\right)$

where $\omega$ is a constant.

3. Active control after completion of the stage

This stage is introduced to adjust the angle of attack. The ship maintains the angle of attack of the T-foil while it continues sailing until time $t_{2}$.
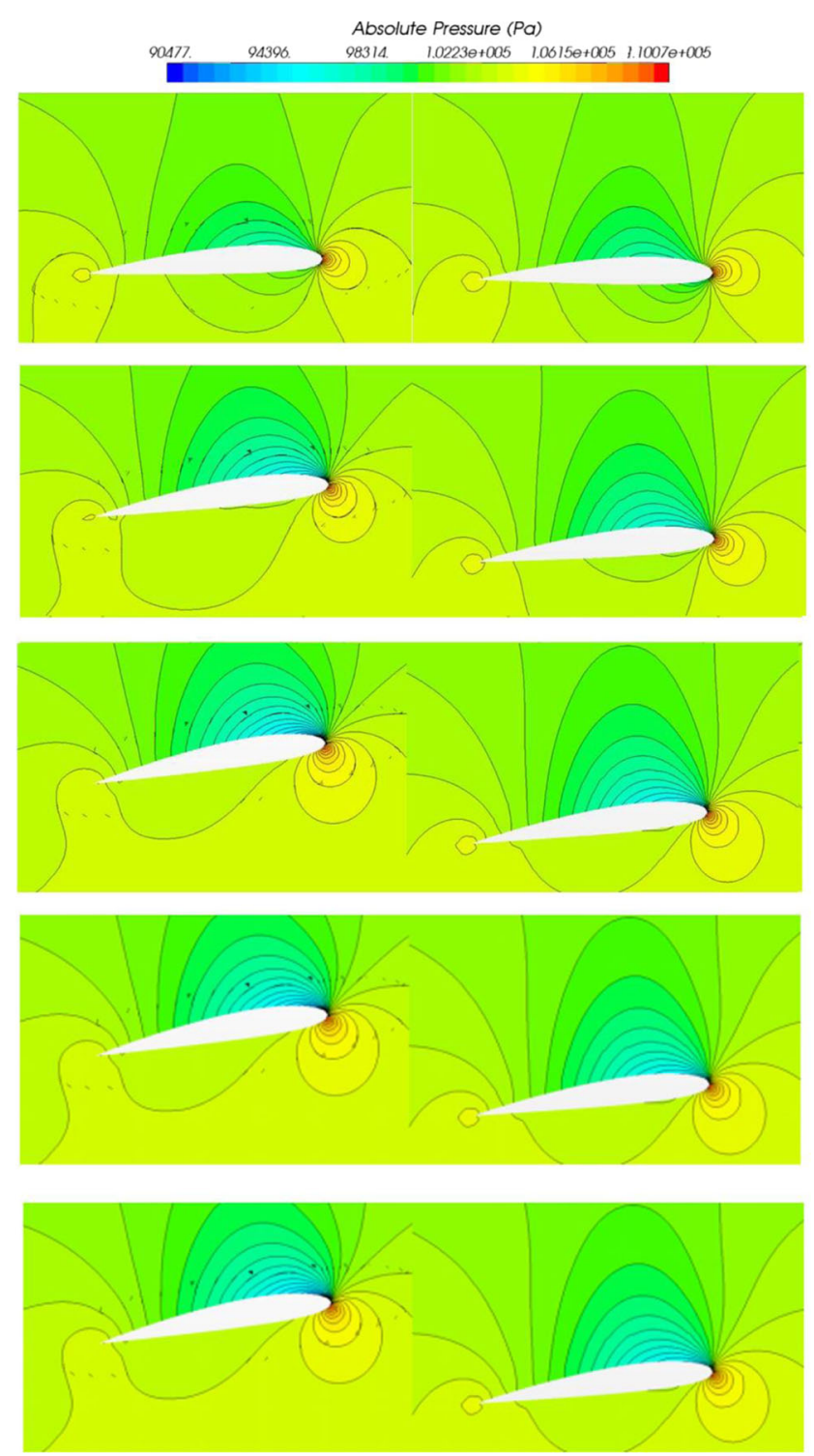

(a) Overlapping mesh

(b) Deforming mesh

Fig. 12 Pressure around the T-foil. a Overlapping mesh. b Deforming mesh 


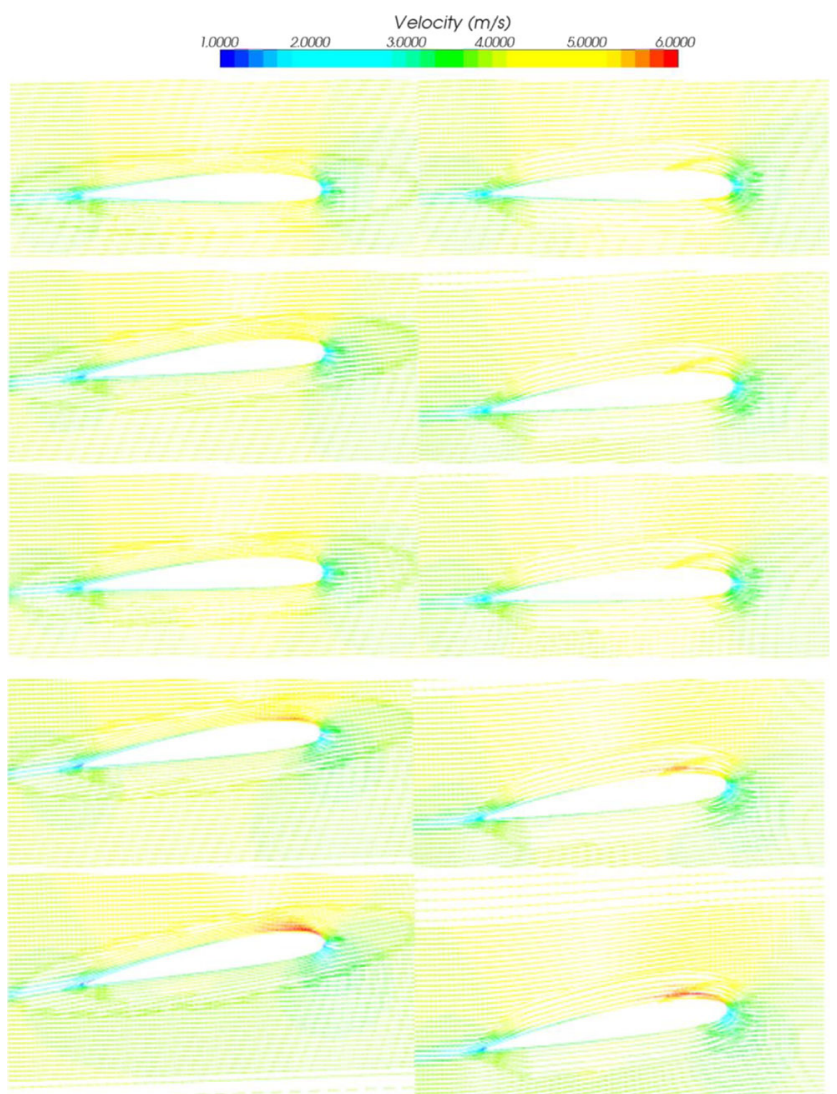

(a) Overlapping mesh

(b) Deforming mesh

Fig. 13 Velocity vector around T-foil. a Overlapping mesh. b Deforming mesh

\subsection{Simulation Results and Analysis}

\subsubsection{Flow Field around T-Foil}

Here, we explore the real changes of the flow field while the Tfoil is rotated. The pressure field and velocity field around the $\mathrm{T}$-foil moving with the ship have been analyzed in the literature (Zhao et al. 2011). In the present study, we examined an upward swing to illustrate the behavior. The motion of the Tfoil was also used to simulate the active swing of the

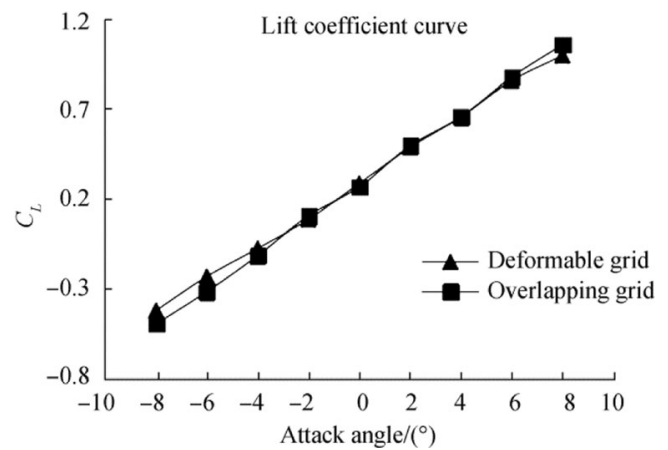

Fig. 14 Dependence of the coefficient of lift on angle of attack for the Tfoil
Table 1 T-foil resistance test data and CFD values

\begin{tabular}{lrrrr}
\hline Fr & Angle of attack/( $\left.{ }^{\circ}\right)$ & EFD/N & CFD/N & Error value/\% \\
\hline 2.83 & 8 & 91.86 & 88.93 & 3.20 \\
& 0 & 85.36 & 83.41 & 2.30 \\
3.03 & -8 & 92.37 & 93.45 & -1.20 \\
& 8 & 115.89 & 112.22 & 3.20 \\
& 0 & 108.47 & 106.82 & 1.50 \\
3.18 & 8 & 116.39 & 117.24 & -0.70 \\
& 0 & 123.62 & 120.69 & 2.40 \\
3.41 & -8 & 115.11 & 113.54 & 1.40 \\
& 8 & 125.19 & 125.93 & -0.60 \\
& 0 & 133.43 & 130.86 & 1.90 \\
3.56 & 8 & 125.19 & 123.66 & 1.20 \\
& 0 & 137.59 & 138.32 & -0.50 \\
& -8 & 142.35 & 140.01 & 1.60 \\
& & 133.31 & 131.48 & 1.40 \\
& & 146.15 & 146.99 & -0.60 \\
\hline
\end{tabular}

hydrofoil. When the active motion of the T-foil was complete, the hull could move in six degrees of freedom. Figures 12 and 13 , respectively, show the pressure and speed around the T-foil in the flow field, with the column of panels showing the evolution in (a) the overlapping grid where motion is considered and (b) the deforming grid where motion is not considered.

By observing the angle of attack and the location of the Tfoil in the figures, we can draw various conclusions. In (a), during active control of the T-foil, the simulated overlapping grid method was used, and in addition to the rotational rigid movement of the T-foil, the T-foil was also dynamically coupled with the hull. In (b), only a swinging of the T-foil can be seen. The position of the T-foil's center of rotation did not change. In regard to the velocity vector, we found that along with the T-foil's upward swing, the angle of attack increased, and the rate of flow of the T-foil surface increased, while the lower surface of water flowed more gradually. Moreover, considering the pressure distribution, the surface of the front T-foil gradually produced a low pressure area. When the angle of attack reached $8^{\circ}$, the low pressure area became apparent. The upper and lower surfaces of the T-foil slowly generated a pressure difference, which led to the generation of a lift. As shown in (a) and (b) of the figures, the hydrodynamic features in both the overlapping mesh and deforming mesh were the same, except that the T-foil motion and the superposition of the hull caused a displacement of the center of the T-foil rotation. Although the position of the T-foil in the flow field was slightly different, this displacement was small, and hence, there did not appear to be much difference in hydrodynamic values between (a) and (b). This agrees with actual situations.

The coefficients of lift for the T-foils obtained by the two methods are compared in Fig. 14. The sinkage and trim are considered in the overlapping grids. Here, the influence of whether to consider the impact of the ship's motion can been 


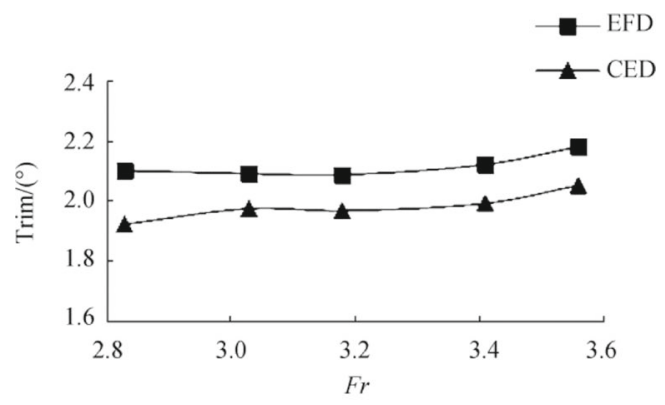

(a) Angle of attack: $8^{\circ}$

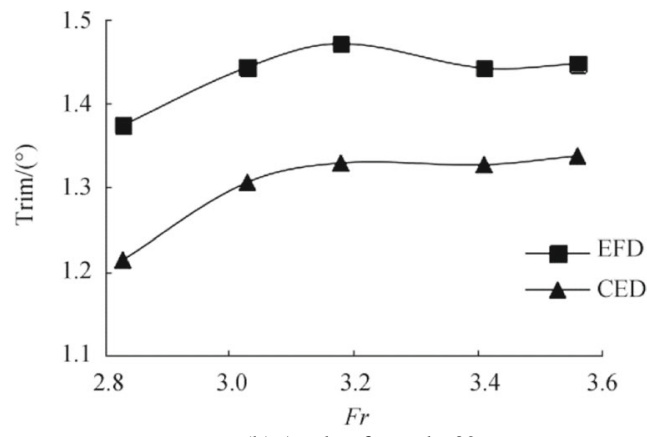

(b) Angle of attack: $0^{\circ}$

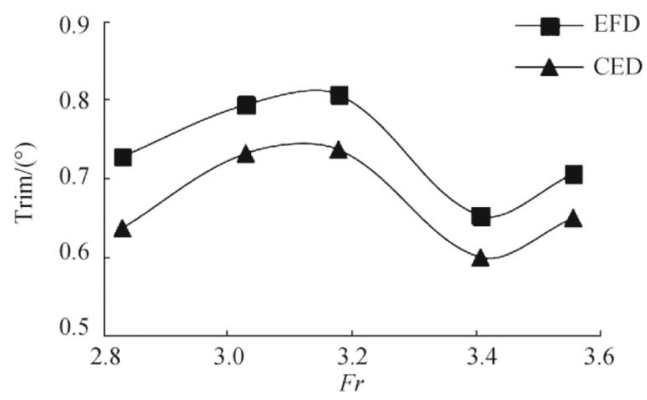

(c) Angle of attack: $-8^{\circ}$

Fig. 15 Calculated and experimental values of trim at different angles of attack. a Angle of attack $8^{\circ}$. b Angle of attack $0^{\circ}$. c Angle of attack $-8^{\circ}$

seen from the change in the trim. In the overlapping grid method, the angle of attack of the T-foil in the geodetic coordinate system needs to be added to the trim between the ship and the T-foil, and this leads to the absolute value for the angle of attack of the T-foil being higher than that when the ship's motion was not considered.

\subsubsection{Navigation State and Hull Resistance}

Table 1 shows the experimental data and numerical results, as well as the deviation in form of percentage error. The hydrodynamic function of the T-foil is to adjust the attitude of the ship by generating a lift. Therefore, during active control of the T-foil, the response of the ship's attitude to an active swing of the T-foil is the focus in this section. The overlapping grid method was applied to calculate the ship's motion during active swinging, and the time history of the

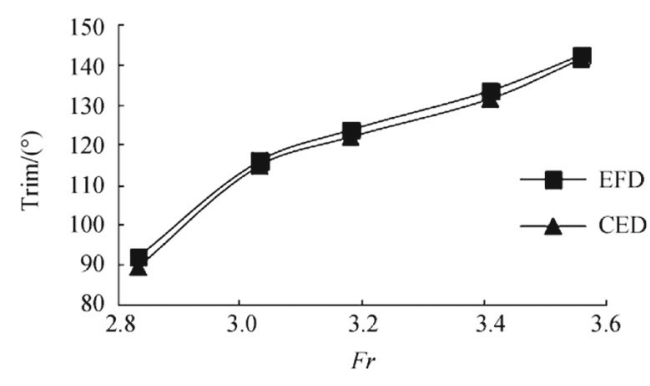

(a) Angle of attack: $8^{\circ}$

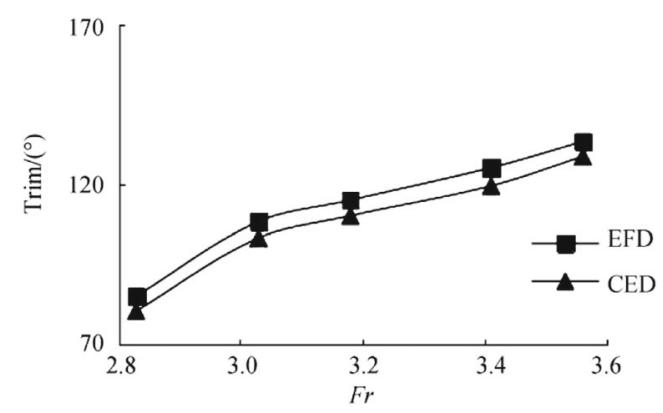

(b) Angle of attack: $0^{\circ}$

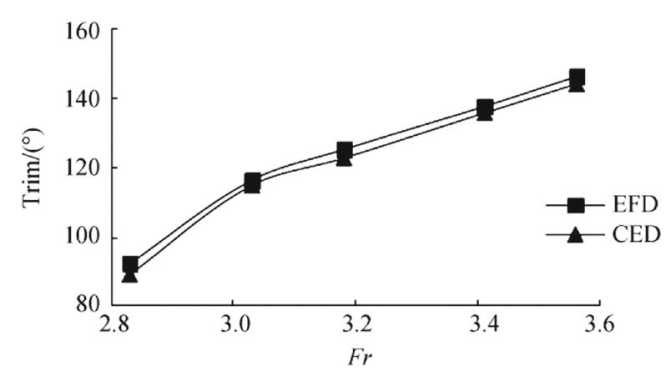

(c) Angle of attack: $-8^{\circ}$

Fig. 16 Calculated and experimental values of total resistance at different angles of attack. a Angle of attack $8^{\circ} \mathbf{b}$ Angle of attack $0^{\circ}$. c Angle of attack $-8^{\circ}$

ship's attitude was obtained, which indicates the ship's response. Calculated values (CFD) and experimental values (EFD) of trim at angles of attack of $8^{\circ}, 0^{\circ}$, and $-8^{\circ}$ are shown in Fig. 14.

Calculated values (CFD) and experimental values (EFD) of the trim and the total resistance at different angles of attack of $8^{\circ}, 0^{\circ}$, and $-8^{\circ}$ (Fig. 15 and Fig. 16 separately) show that a better agreement was obtained using overlapping grid method (CFD).

As shown in Figs. 15 and 16, when the overlapping grid method was used, only a small difference existed between the calculated and experimental values of the attitude response and resistance at different angles of attack of the T-foil. This indicates that the response of the ship's sailing attitude to the movement of the active appendages calculated using this method is accurate and reliable, and that this overlapping grid technique is effective for numerically simulating rigid boundary movements. 


\section{Conclusions}

The movement of an active T-foil was simulated using an overlapping grid technique. By simulating an active T-foil and hull using this technique and determining the ship's attitude during active T-foil swinging, the ship's response could be analyzed and the change in total resistance could be obtained. From the moment the active T-foil stopped moving, there was no change in the ship's sailing attitude, indicating that the response was in real time. In addition, by comparison, we found that the test values and calculated values of trim and total resistance were in good agreement, indicating the accuracy, feasibility, and effectiveness of the overlapping grid technique.

Funding Supported by the National Natural Science Foundation of China (Grant No. 51509053).

Open Access This article is distributed under the terms of the Creative Commons Attribution 4.0 International License (http:// creativecommons.org/licenses/by/4.0/), which permits unrestricted use, distribution, and reproduction in any medium, provided you give appropriate credit to the original author(s) and the source, provide a link to the Creative Commons license, and indicate if changes were made.

\section{References}

Chen QR, Ye HK, Guan YM (2010) Hydrodynamic analysis of 3-D hydrofoil under free surface in time domain. J Ship Mech 14(12): 1331-1339

Deng R, Huang DB, Zhou GL (2014) Research on the influence of T-foil on the hydrodynamic performance of trimaran. The Twenty-fourth International Ocean and Polar Engineering Conference, Busan. $758-763$,

Esteban S, Giron-Sierra JM, Andres-Toro BD, Dela Cruz JM (2005) Fast ships models for seakeeping improvement studies using flaps and Tfoil. Math Comput Model 41(1):1-24. https://doi.org/10.1016/j. mcm.2004.09.002

Feng WL, Li J, Zhang W (2008) Numerical simulation of airfoil icing based on deformation grid. J Northwest Polytech Univ 26(5):550555 (in Chinese)

Hao HB, Guo ZQ, Ma QW, Dai SS (2015) A preliminary study on the hydrodynamic propulsive force of a pair of inversely oscillating hydrofoils. The twenty-fifth international offshore and polar engineering conference. 1040-1045, Kona, Hawaii, USA

Ji HT, Chen JR, Li W (2006) Several measures of ship aft modification. China Ship Res 1(3):41-46 (Journals in Chinese)

Jiang Y, Sun HB, Zou J, Yang DM, Hu AK (2016) Influence of anglevariable stern flap on resistance performance of stepped planing hull. J Shanghai Jiaotong Univ 320-325(in Chinese):3. https://doi. org/10.16183/j.cnki.jsjtu.2017.03.012
Jin MX, Zhang JN, Zhang L, Jin MY (2015) Influence of speedboat stern pressure plate on hydrodynamic performance of speedboat. China Water Transp 07:9-10 (in Chinese)

Kehoe JW, Brower KS, Meier HA, Runnerstro CE (1983) U. S. and foreign hull form, machinery, and structure design practices. Nav Eng J 95(6):36-53. https://doi.org/10.1111/j.1559-3584.1983. tb01681.x

Liang H (2015) Lifting theories for wing-in-ground effect and hydrofoils in the vicinity of a free surface. $\mathrm{PhD}$ thesis, Dalian University of Technology, Dalian. 23-25

Liang LH, Jiang JL, Zhang ST (2013) Improving the vertical motion of wave piercing catamaran using T-foil. ICMA 2013 (8):1481-1485. https://doi.org/10.1109/ICMA.2013.6618132

Liu YH (2014) The research of T-foil and flap's effect on wave-piercing catamaran seakeeping. Master thesis, China Ship Research Institute, Beijing. 25-30

Liu AL (2016) The influence of spray rails and stern flat on planning hull resistance performance. Master thesis, Harbin Engineering University, Harbin, 25-43

Liu YH, Wang GY, Wang Q, Chai X, Ren WL (2016) Influence of T-foil on wave resistance of wave-piercing catamaran. China Water Transp 2:12-13 (in Chinese)

Ma C (2012) The effect of interceptor stern flap on the resistance of planning boat. Master thesis, Harbin Engineering University, Harbin: 41-59

Ning P (2017) Prediction of hydrodynamic performance on threedimensional hydrofoil and propeller using potential panel method. Master thesis, Zhejiang University, Zhejiang, 47-60

Nirschl H (2005) Overlapping grids for the simulation of particle interaction at the micro scale. China Particuol 3(1-2):60-67

Piperno S (1997) Explicit/implicit fluid/structured staggered procedures with a structural prediction and fluid subcycling for 2D inviscid aeroelastic simulation. Int J Numer Meth Fluids 25:1207-1226

Shao SM, Wang YC (1981) The effects of stern trimming flap on resistance of high speed craft. Shipbuild China 01:29-39 (in Chinese)

Si HL, Yu H, Li ZJ, Geng YC, Hu JJ (2015) The research of slamming pressure impacted on wave suppression board. Ship Sci Technol 02: 19-23 (in Chinese)

Wang TC (2017) Research on hydrodynamic characteristics of an oscillating foil near free surface. Master thesis, Harbin Engineering University, Harbin. 42-65

Yang Q (2012) Research on the design of T-foil for trimaran. Master thesis, Harbin Engineering University, Harbin 23-25

Yang Q, Lin Z, Guo ZQ (2013) Theoretical analysis and simulation on anti-rolling effect of trimaran fitted $\mathrm{T}$ foil. J Cent South Univ (Sci Technol) 44(2):46-51 (in Chinese)

Yang JL, Yang DM, Li P, Lin Z (2017) Numerical analysis of peak resistance reduction by bubbly layer for surface effect ship. J Harbin Eng Univ 38(11):1709-1714. https://doi.org/10.11990/ jheu.201608049

Zhang LP, Deng XG, Zhang HX (2007) Moving mesh generation and unsteady calculation methods study. Adv Mech 40(4):424-447 (in Chinese)

Zhao FM, Gao CJ, Xia Q (2011) Overlap grid research on the application of ship CFD. J Ship Mech 15(4):332-341 (in Chinese) 